\title{
Performance d'un dispositif amélioré de séchage de poisson fermenté appelé lanhouin au Bénin
}

\author{
Pierre DOSSOU-YOVO ${ }^{1 *}$, Innocent BOKOSSA ${ }^{2}$, Hélène AHOUANDJINOU ${ }^{1}$, \\ Svetlana ZOLOTOKOPOVA ${ }^{3}$ et Iraida ALAGUINA ${ }^{3}$ \\ ${ }^{1}$ Laboratoire de Recherche en Traitement et Conservation des Produits \\ ${ }^{2}$ Halieutiques, FAST/ Université d'Abomey-Calavi, Bénin \\ ${ }^{3}$ Laboratoire de Microbiologie et de Technologie Alimentaire, FAST/ Université 'Abomey-Calavi, Bénin \\ Université Technique d'Etat d'Astrakhan, Russie \\ *Auteur correspondant ; E-mail : pidam57@yahoo.fr ; pierre.dossouyovo@uac.bj, BP 1270 Abomey-Calavi, \\ Bénin. Tél.: (229) 93797239.
}

\section{RESUME}

Le séchage des aliments en Afrique est sujet à d'énormes contraintes dues au faible niveau de développement industriel et technologique. Le séchage est en général naturel et utilise du matériel rudimentaire. Dans ce travail, il a été conçu un nouveau matériel de séchage du poisson fermenté produit au Bénin. Ce produit appelé lanhouin est du poisson fermenté salé et mis à sécher dans des conditions très insalubres. Il a été étudié la performance du dispositif amélioré conçu pour le séchage du lanhouin dans les conditions naturelles de l'environnement de production. La température ambiante variait de $30{ }^{\circ} \mathrm{C}$ les matins à $27{ }^{\circ} \mathrm{C}$ en moyenne les soirs. Le dispositif de séchage inventé protège le poisson fermenté des infestations. Les poissons séchés par ce dispositif présentent une texture plus ferme sans odeur agressive et possèdent une humidité relative assez basse (42,64\%) pour être conservés plus longtemps.

() 2010 International Formulae Group. All rights reserved.

Mots clés: Matériels rudimentaires, nouveau matériel, infestations, texture ferme, humidité relative.

\section{INTRODUCTION}

Le poisson est un aliment très important du fait de sa valeur nutritionnelle. Cependant c'est une denrée hautement périssable, en particulier dans les zones tropicales à climat chaud où les infrastructures d'écoulement du poisson frais n'existent pas toujours. Pour palier à ce problème, une partie de la prise de poisson est transformée pour assurer sa conservation. Il existe plusieurs techniques traditionnelles de conservation du poisson frais, dont la fermentation artisanale pour la production du lanhouin (Tohouégnon et al.,
1997) qui est du poisson fermenté, salé et séché. La matière première est constituée de six espèces fréquemment utilisées pour fabriquer le lanhouin. Ce sont les Scombridae, les Clupidae, les Scianidae, les Polynemidae, les Carangidae et les Ariidae. Les Polynemidae sont les plus utilisés pour la production du lanhouin. L'espèce la plus rencontrée durant les investigations, est le Galeoides decadactylus, appelé faux capitaine.

Le séchage du lanhouin demeure encore traditionnel au Bénin à l'instar des pays africains où le soleil brille la majeure partie de 
l'année. Mais le séchage tel que pratiqué dans les pays en voie de développement est à l'origine de pertes importantes et aboutit à un produit de qualité sanitaire médiocre (Essuman, 1992). Il ne peut d'ailleurs en être autrement car les poissons mis à sécher sont exposés aux insectes (Essuman, 1992), vecteurs des microorganismes, et aux margouillats. Par ailleurs les ouvrières, exposées au soleil, doivent retourner les poissons une à deux fois dans la journée avec des mains qui ne sont pas toujours propres.

Le présent article contribue à l'amélioration de la qualité du lanhouin à travers la mise au point et l'utilisation d'un dispositif amélioré et pratique de séchage du lanhouin. Pour en arriver là, il a fallu mesurer la performance de séchage du dispositif amélioré inventé en comparaison avec les résultats obtenus par un séchage artisanal.

\section{MATERIEL ET METHODES}

Une enquête a été réalisée dans les départements du Littoral et du Mono. Le choix de ces départements se justifie par le fait que le lanhouin est un condiment utilisé par les populations Xwla et Mina qui y vivent. Cette enquête a permis de connaître les différentes variantes de la technologie d'obtention du poisson fermenté et les caractéristiques du matériel artisanal de séchage utilisé. Pour cela, une méthode d'échantillonnage a été adoptée ; celle de l'échantillonnage en grappes à deux degrés d'unités : au niveau primaire, les villes enquêtées et au niveau secondaire, les sites de séchage de poissons fermentés.

Au niveau primaire, les unités ont été choisies en raison de la prépondérance de la population productrice du poisson fermenté dans les villes de Cotonou (Akpakpa-dodomé) et de Grand-Popo (Ayi-guinnou et Gbèkon), du fait de la proximité de l'océan pourvoyeur des poissons traités. Au niveau secondaire, les sites de séchage ont été choisis de façon aléatoire tant dans le Littoral (Cotonou) que dans le Mono (Grand-Popo).

Les productrices ont été choisies de manière aléatoire dans les zones identifiées. Au total 9 sites de production ont été visités ; 27 productrices ont été questionnées, à raison de 3 productrices par site enquêté.
Des entretiens ont été réalisés à l'aide d'un questionnaire puis les données recueillies ont été traitées par le logiciel Winstat.

Pour caractériser les lanhouin selon les deux types de dispositif de séchage, 30 échantillons de lanhouin fabriqués à base de Pseudotolithus senegalensis ont été collectés sur 3 sites de production artisanale (lot 1), et 30 échantillons selon la technologie améliorée (lot 2) proposée par Dossou-yovo (2002). Le lot1 est séché avec un dispositif artisanal (van surélevé à l'aide d'un panier) tandis que le lot 2 est séché avec le dispositif amélioré de séchage. Différentes températures ont été relevées au cours du séchage.

Le $\mathrm{pH}$ des échantillons a été mesuré à l'aide d'un pH-mètre PICCOLO ATC; les teneurs en sel, en eau et en protéines ont été déterminées respectivement par les méthodes AOAC N ${ }^{\circ}$ 937.09, 950.46, 981.10 (1995); la teneur en azote basique volatil total (ABVT) a été déterminée par la méthode décrite par Martin (1979); les matières grasses ont été déterminées selon Pearson (1976) ; les germes aérobies mésophiles, les moisissures et levures, les anaérobies sulfitoréducteurs, les staphylocoques, les coliformes, les streptocoques et les salmonelles ont été énumérés, respectivement, selon les normes ISO 4833, ISO 7954, ISO 688-1, ISO 7252, ISO 4831, ISO 7899-2, ISO 6579.

\section{Dispositifs de séchage Dispositif artisanal}

Le dispositif artisanal est constitué de nattes tressées à l'aide de nervures d'écorces de palmier ou de cocotier disposées sur des pieux de 1 à 1,20 $\mathrm{m}$ de haut au dessus du sol. Aussi on rencontre des vans disposés au dessus de paniers de 40 à $50 \mathrm{~cm}$ de haut et sur lesquels sont étalés les produits (lanhouin) à sécher.

\section{Dispositif amélioré}

Le dispositif amélioré de séchage est réalisé dans le centre de menuiserie de l'Ecole Polytechnique d'Abomey-Calavi (EPAC). Il est en bois massif avec grillage inoxydable, le tout pouvant pivoter sur des tréteaux.

Ce dispositif est composé :

- d'une caisse grillagée ; d'un tiroir grillagé ; 
- de deux traverses de support ; de quatre (4) tréteaux ; de deux (2) poignets arrondis ; de chevilles de blocage.

\section{La caisse grillagée}

Elle est de forme rectangulaire de $2 \mathrm{~m}$ de long, de $1 \mathrm{~m}$ de large et de $0,1 \mathrm{~m}$ de hauteur avec une feuillure.

Il y est aménagé sur les deux largeurs et une longueur, une feuillure devant accueillir le tiroir coulissant. La feuillure a une largeur de $25 \mathrm{~mm}$ et une profondeur de $1,5 \mathrm{~mm}$. A la base de la caisse, est fixé du grillage inoxydable à mailles $2 \mathrm{~mm}^{2}$. Le bois assemblant la caisse a une épaisseur de $35 \mathrm{~mm}$ sur $100 \mathrm{~mm}$ de profondeur. La caisse est à l'équerre sur deux angles. Elle sert de barrière pour les mouches et autres insectes volants.

\section{Le tiroir grillagé}

Le tiroir est un assemblage de bois de traverses. Il a une forme rectangulaire de 1,96 $\mathrm{m}$ de longueur et de $0,97 \mathrm{~m}$ de large. Les traverses à chaque extrémité sont de dimensions $9,5 \times 2,0 \mathrm{~cm}$ et deux traverses de $4,0 \times 2,0 \mathrm{~cm}$ au milieu du tiroir pour servir de support au grillage. Le grillage est fixé dans une feuillure usinée et condamné par des couvre-joints.

\section{La traverse}

La traverse à queue d'aronde est du bois d'une longueur de $114 \mathrm{~cm}$, d'une épaisseur de $2,5 \mathrm{~cm}$ et d'une largeur de $6,5 \mathrm{~cm}$. Elle traverse une entaille de segments de bois reliant les deux pieds du tréteau doté de queue d'aronde à chaque extrémité pour la consolidation des pieds.

\section{Le tréteau}

Le tréteau est constitué de deux pieds dont les dimensions sont les suivantes : hauteur $=120 \mathrm{~cm}$, épaisseur $=2,5 \mathrm{~cm}$ et la largeur de chacun des pieds est de $6,5 \mathrm{~cm}$ La tête du tréteau est constituée d'entailles à queue d'aronde accueillant une traverse au milieu de laquelle est aménagée une entaille de support arrondie à la base. Dans cette entaille passe un poignet arrondi fixé à la caisse pour la faire pivoter.

\section{Les chevilles}

Les chevilles sont des segments de bois taillés et arrondis qui servent au système de blocage et de maintien en équilibre du dispositif.

\section{RESULTATS \\ Conditions de séchage}

Les Figures 1, 2, 3 et 4 présentent les différents stades d'utilisation du dispositif de séchage. Les Figures $5 ; 6 ; 7$ montrent les conditions du séchage artisanal à Akpakpadodomè. Celles numérotées de 8 à 10 montrent l'insalubrité de l'environnement de séchage au lieu sus indiqué, et les Figures 11 et 12 présentent l'environnement de séchage du lanhouin à Ayi-guinnou (Grand-Popo). Les Figures 13 et 14 présentent les poissons séchés à l'aide du dispositif proposé dans le cadre de l'amélioration des conditions d'hygiène et de salubrité dans cette activité de production du poisson fermenté, salé et séché au Bénin.

Les différentes températures au cours du séchage sont consignées dans le Tableau 1 ; les résultats du Tableau 1 montrent une élévation graduelle de température au cours de la matinée, laquelle température ne chute que légèrement dans l'après-midi (soir à 18h), donnant une moyenne de $28{ }^{\circ} \mathrm{C}$ les soirs pour les trois jours d'expérimentation.

\section{Analyses microbiologiques et physico- chimiques}

Les Tableaux 2 et 3 présentent respectivement les résultats des analyses microbiologiques, et physico-chimiques. Le Tableau 2 expose l'état microbiologique des produits artisanaux et améliorés. Ceci saute à l'œil par la présence ou non de microorganismes indésirables dans l'un ou l'autre produit. Il est à constater l'absence de levures, de moisissures, de coliformes totaux et fécaux, d'E. coli, de Staphylocoques aureus, de salmonelles et d'anaérobies sulfitoréducteurs dans le poisson fermenté salé et séché par le matériel amélioré. A l'opposé, le poisson fermenté salé et séché par le matériel artisanal présente l'absence de levures, d'E. coli, de Staphylocoques aureus et de salmonelles. Le Tableau 3 fait constater une humidité relative d'environ $42 \%$ du poisson fermenté amélioré, opposée à celle du poisson fermenté artisanal. 
Tableau 1 : Durée et température de séchage au mois d'octobre correspondant au pic de la pêche du Galeoides decadactylus.

\begin{tabular}{llccc}
\hline $\begin{array}{l}\text { Durée de } \\
\text { séchage }\end{array}$ & $\begin{array}{l}\text { Différents } \\
\text { moments de la } \\
\text { journée }\end{array}$ & $\begin{array}{c}\text { Température } \\
\text { ambiante }\left({ }^{\circ} \mathbf{C}\right)\end{array}$ & $\begin{array}{c}\text { Température à l'intérieur } \\
\text { du dispositif de séchage } \\
\left({ }^{\circ} \mathbf{C}\right)\end{array}$ & $\begin{array}{c}\text { Température sur } \\
\text { le grillage du } \\
\text { dispositif de } \\
\text { séchage }\left({ }^{\circ} \mathbf{C}\right)\end{array}$ \\
\hline Premier jour & Matin & $30,2 \pm 0,6$ & idem & idem \\
& Midi & $33,8 \pm 0,1$ & $33,5 \pm 0,1$ & $36,4 \pm 0,1$ \\
& Soir & $30,0 \pm 0,7$ & $30,2 \pm 0,7$ & $30,7 \pm 0,7$ \\
\hline Deuxième jour & Matin & $29 \pm 0,8$ & idem & Idem \\
& Midi & $32,8 \pm 0,2$ & $35,5 \pm 0,2$ & $35,8 \pm 0,2$ \\
& Soir & $29,1 \pm 0,5$ & $29,1 \pm 0,5$ & $29,5 \pm 0,5$ \\
\hline Troisième jour & Matin & $30,2 \pm 1,0$ & idem & $38,0 \pm 0,2$ \\
& Midi & $33,6 \pm 0,2$ & $37,8 \pm 0,2$ & $31,0 \pm 0,4$ \\
\hline
\end{tabular}

Tableau 2 : Analyses microbiologiques.

\begin{tabular}{lcc}
\hline Earamètres & $\begin{array}{c}\text { Poisson fermenté } \\
\text { salé et séché } \\
\text { artisanal }\end{array}$ & $\begin{array}{c}\text { Poisson fermenté salé } \\
\text { et séché amélioré }\end{array}$ \\
\hline Germes aérobies mésophiles & $6,8.10^{9} \mathrm{UFC} / \mathrm{g}$ & $4,3.10^{5} \mathrm{UFC} / \mathrm{g}$ \\
Levures & Absence & Absence \\
Moisissures & $90 \mathrm{UFC} / \mathrm{g}$ & Absence \\
Coliformes totaux & $9 \mathrm{UFC} / \mathrm{g}$ & Absence \\
Coliformes fécaux & $9 \mathrm{UFC} / \mathrm{g}$ & Absence \\
E. coli & Absence & Absence \\
Streptocoques & $200 \mathrm{UFC} / \mathrm{g}$ & $150 \mathrm{UFC} / \mathrm{g}$ \\
Staphylococcus. aureus & Absence & Absence \\
microcoques & $1.4 .10^{3} \mathrm{UFC} / \mathrm{g}$ & $1.10^{3} \mathrm{UFC} / \mathrm{g}$ \\
Salmonelles & Absence & Absence \\
Anaérobies & $550 \mathrm{UFC} / 20 \mathrm{~g}$ & Absence \\
Sulfito-réducteurs & &
\end{tabular}

Tableau 3: Analyses physico-chimiques et biochimiques.

\begin{tabular}{lcc} 
Paramètres & $\begin{array}{c}\text { Poisson fermenté salé et séché } \\
\text { artisanal }\end{array}$ & $\begin{array}{c}\text { Poisson fermenté salé et } \\
\text { séché amélioré }\end{array}$ \\
\hline Humidité (\%) & $51,08 \pm 0,37$ & $42,60 \pm 0,18$ \\
Matière sèche (\%) & $48,92 \pm 0,19$ & $57,40 \pm 0,32$ \\
pH & $6,46 \pm 0,07$ & $6,45 \pm 0,02$ \\
ABVT (mg N/ 100g) & $163,8 \pm 0,09$ & $169,4 \pm 0,01$ \\
Protéines (\%) & $50,57 \pm 0,05$ & $53,09 \pm 0,11$ \\
Matières grasses (\%) & $2,65 \pm 0,03$ & $2,87 \pm 0,02$ \\
Salinité (g/ $/ 100 \mathrm{~g})$ & $16,85 \pm 0,01$ & $20,65 \pm 0,04$ \\
\hline
\end{tabular}




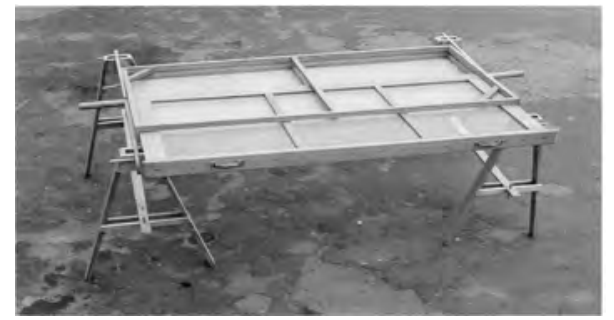

Figure 1 : Ouverture de la caisse.

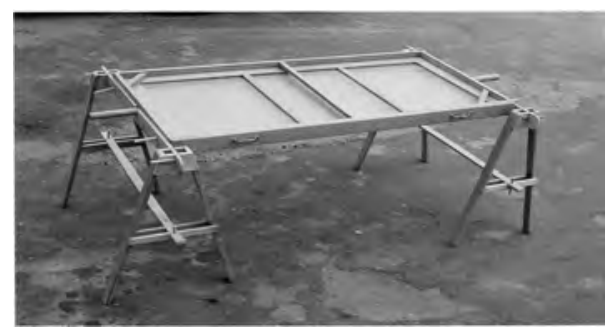

Figure 2 : Caisse fermée.

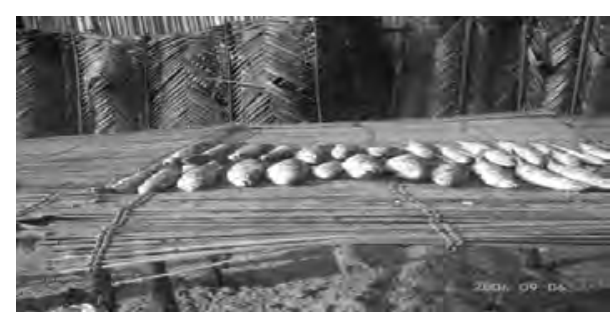

Figure 5: Aspect d'un séchage de lanhouin à Akpakpa-dodomè.

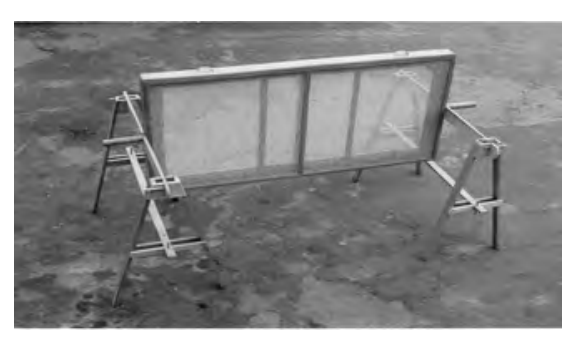

Figure 3 : Pivotement de la caisse.

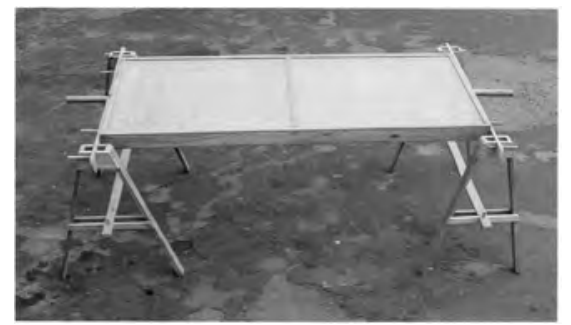

Figure 4 : Caisse pivotée.

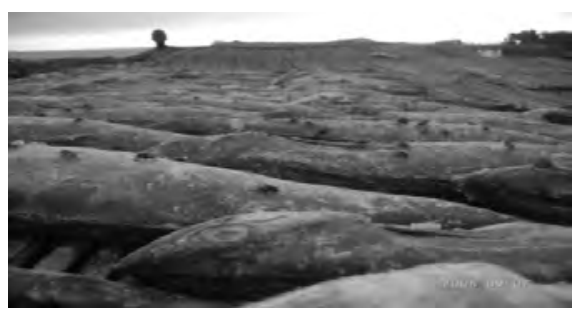

Figure 6 : Le lanhouin est envahi par les mouches.

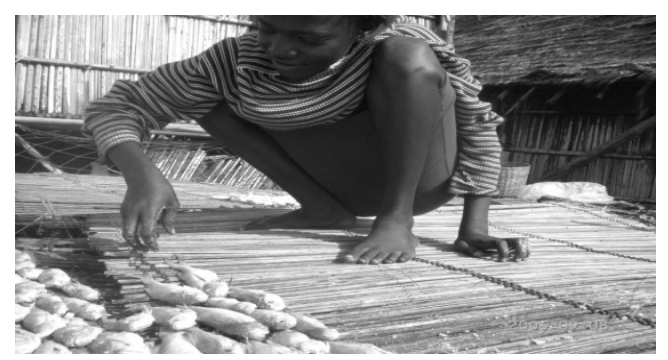

Figure 7 : Pratique non hygiénique du séchage à Akpakpa-dodomè. 

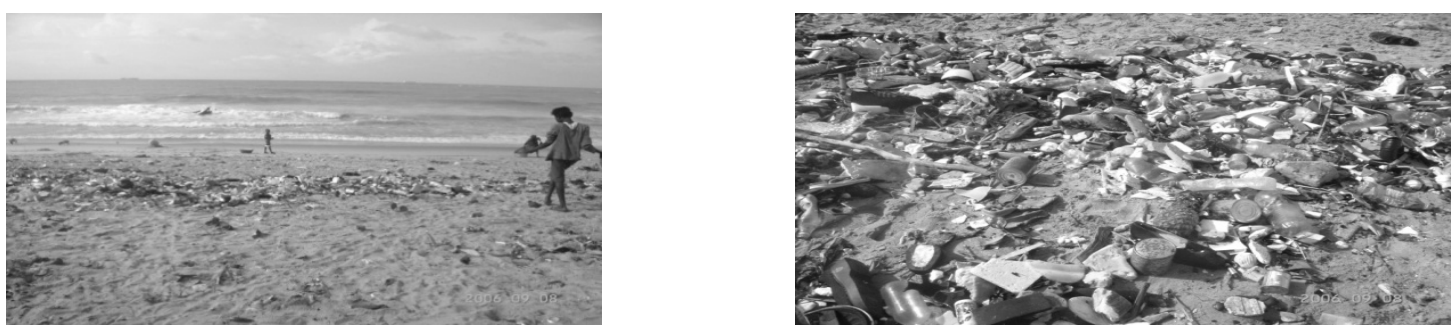

Aspect de l'environnement insalubre à Akpakpa-dodomè Figure 8

Figure 9

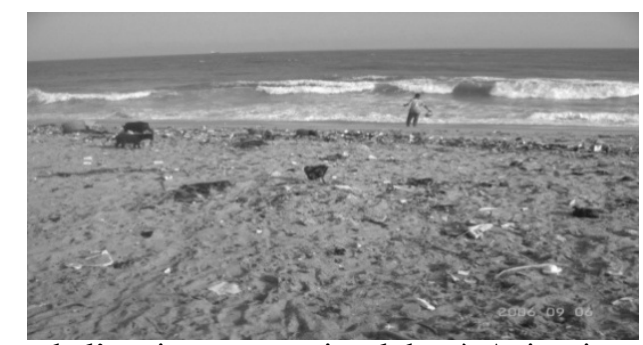

Aspect de l'environnement insalubre à Ayi-guinnou

Figure 10
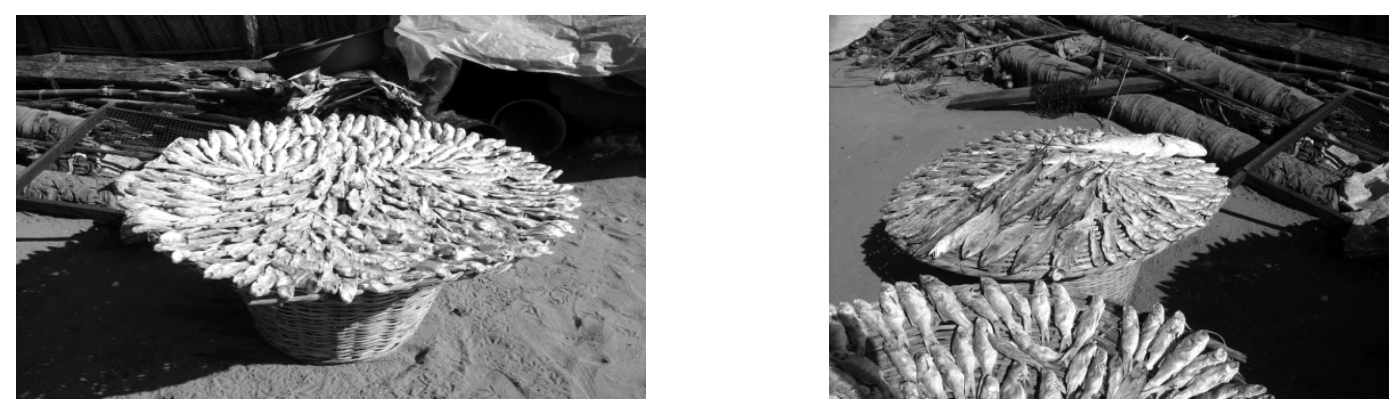

Figure 11

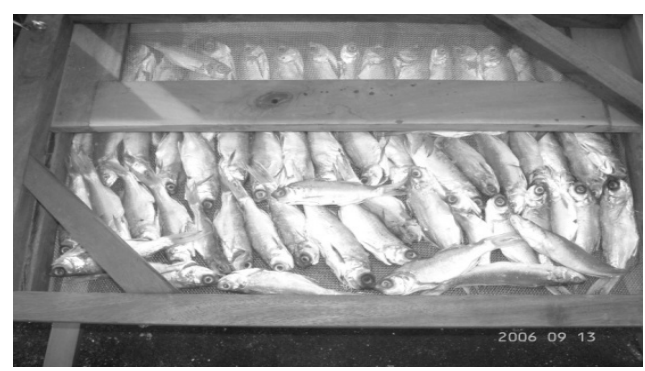

Séchage du lanhouin à l'aide du dispositif amélioré
Séchage de lanhouin à Ayi-guinnou

Figure 12

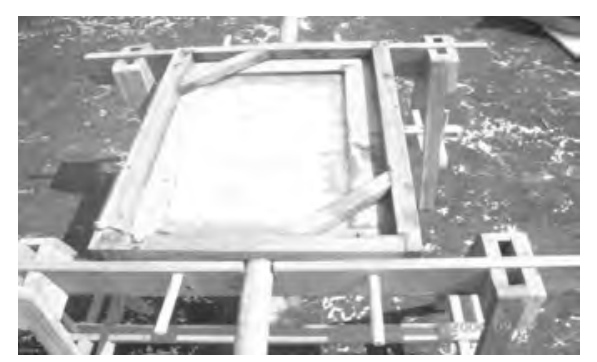

Figure 14 


\section{DISCUSSION}

Les résultats consignés dans le Tableau 1 montrent que les températures ambiantes observées dans la matinée au mois d'Octobre 2006 sont presque constantes avec une légère fluctuation (29 à $\left.30,2{ }^{\circ} \mathrm{C}\right)$. A la mi-journée (midi) cette température est d'environ $34{ }^{\circ} \mathrm{C}$ alors que dès 18 heures elle peut chuter jusqu'à $27{ }^{\circ} \mathrm{C}$. Nous avons constaté que le grillage du dispositif de séchage se réchauffe les midis jusqu'à $36{ }^{\circ} \mathrm{C}$ au minimum et $38{ }^{\circ} \mathrm{C}$ au maximum. Par contre les soirs, elle chute jusqu'à $29{ }^{\circ} \mathrm{C}$. A l'intérieur du dispositif, la température a fluctué les midis entre $33{ }^{\circ} \mathrm{C}$ et $38{ }^{\circ} \mathrm{C}$. Dans cette même enceinte du dispositif cette température a fluctué les soirs entre $29^{\circ} \mathrm{C}$ et $30{ }^{\circ} \mathrm{C}$. Pour le séchage des poissons fermentés et salés de type amélioré, l'air par convexion transmet la chaleur au produit à travers le grillage inoxydable. En effet, le réchauffement du grillage du dispositif se révèle par les mesures de température prises à l'aide du thermomètre placé au contact de ce dernier, qui a donné les valeurs fortes obtenues à midi (de $36^{\circ} \mathrm{C}$ à $\left.38^{\circ} \mathrm{C}\right)$.

Il va sans dire que les températures, à l'intérieur du dispositif de séchage, très voisines de celles ambiantes, et parfois supérieures, dénotent de l'échange de température entre le produit et le milieu ambiant d'une part, et du transfert de chaleur au poisson par le grillage d'autre part. $\mathrm{Ce}$ phénomène se produisant de façon concomitante permet une accélération dans l'échappement de l'eau libre, sous forme de vapeur, dans l'atmosphère, et la concentration dans le poisson du chlorure de sodium qui y est entré lors du salage par osmose pour réduire la quantité d'eau.

L'analyse des résultats du Tableau 2 fait constater que les germes aérobies mésophiles dénombrés au niveau du lanhouin artisanal et amélioré sont respectivement de $6,8.10^{9}$ et $4,3.10^{5} \mathrm{UFC} / \mathrm{g}$. La flore aérobie mésophile totale (FAMT) dénombrée dans le lanhouin amélioré respecte les normes: FAMT $<5,5.10^{5}$ UFC/g. La FAMT du lanhouin artisanal trouve sa justification dans les conditions insalubres du séchage. En effet, c'est dans un environnement totalement insalubre que s'opère la production du lanhouin. Des pratiques malsaines de populations totalement indigentes offrent un spectacle de désolation quant à la sécurité alimentaire au Bénin. Juste à proximité du lieu de production et de séchage du lanhouin, en l'absence de toilettes, la population se soulage de ses déjections. La flore osmophile constituée de levures et de moisissures est quasi absente au niveau des deux types de poissons, du fait de la nature suffisamment salée de ces derniers. La charge microbienne des indicateurs de pollution fécale (coliformes ou coliformes fécaux) est quasi nulle au niveau du lanhouin amélioré. Ceci explique une absence de contaminations fécales récentes et trouve sa justification dans les moyens de séchage utilisés qui ne facilitent pas des contaminations croisées induites par les mouches et l'environnement. Par contre, on remarque la présence de contamination croisée dans le produit artisanal. Il est à remarquer l'absence d'E. coli dans tous les deux types de lanhouin (Tableau 2). Ce qui peut se justifier par l'absence de contamination fécale de l'eau utilisée pour le traitement des matières premières.

Néanmoins, la présence des streptocoques témoigne d'une contamination ancienne due à l'insalubrité du lieu de pêche des poissons. Notons que le nombre de streptocoques dans l'échantillon artisanal est supérieur à celui retrouvé dans l'échantillon amélioré (200 UFC/g contre $150 \mathrm{UFC/g}$ ). On note une absence totale des anaérobies sulfitoréducteurs (ASR) dans le lanhouin amélioré. La charge microbienne du lanhouin artisanal en anaérobies sulfito-réducteurs est de 550 UFC/20 g. Nous pouvons dire que le dispositif artisanal de séchage ne met pas le lanhouin à l'abri des mouches et du sable qui peuvent être des vecteurs des ASR. Alors que le dispositif amélioré de séchage, constitué d'une caisse grillagée et surélevé au-dessus du sol protège de l'invasion des insectes et des animaux domestiques. Aucun des échantillons analysés ne présente de risque de contamination en salmonella. Ces résultats se rapprochent de ceux d'Anihouvi (2005). 
En conséquence de ce processus de séchage, les résultats des analyses physicochimiques et biochimiques consignés dans le Tableau 3 révèlent que les poissons séchés par le dispositif ont une humidité relative inférieure à celle des échantillons artisanalement séchés : $42,60 \%$ contre $51,08 \%$. Le $\mathrm{pH}$ des échantillons est sensiblement égal à 6,4. Des valeurs similaires ont été rapportées sur le «momone », poisson fermenté ghanéen analogue au lanhouin (Essuman, 1992). Il n'existe pas à notre connaissance dans la littérature un $\mathrm{pH}$ de référence par rapport au lanhouin. La teneur en ABVT dans les deux types de lanhouin est $163,8 \mathrm{mg} \mathrm{N} / 100 \mathrm{~g}$ pour le produit artisanal et $169,4 \mathrm{mg} \mathrm{N} / 100 \mathrm{~g}$ pour le produit amélioré ; la valeur limite maximale étant de 500 mg N/g (Martin, 1979).

\section{Conclusion}

Il ressort de cette étude que le lanhouin amélioré bénéficie d'une hygiène plus prononcée que celui artisanal. Par conséquent, sa consommation et sa conservation seraient plus rassurantes. En outre, le dispositif amélioré de séchage qui a permis l'obtention de lanhouin de bonne qualité sanitaire allège également la tâche aux productrices. Il urge d'intensifier la formation des productrices pour les convaincre des avantages du nouveau matériel de séchage et faciliter son acquisition et son appropriation.

\section{REFERENCES}

Abbey LD, Hodari-Okae M, Osei-Yaw A. 1994. Studies on traditional processing and quality of fermented fish "momone". Food Research Institute, Accra (Ghana).
Anihouvi VB, Hounhouigan DJ, Ayernor G. 2005. Production et commercialisation du « lanhouin », un condiment à base de poisson fermenté du golfe du Bénin. Cahiers d'Etudes et de Recherches Francophones / Agricultures. 14(3): 323330.

AOAC (Association of Official Analytical Chemists). 1995. Official Methods of Analysis (16th edition). Gaithersburg: Maryland, Etats-Unis.

Dossou-Yovo P. 2002. Justification biochimique de l'amélioration des procédés traditionnels de production du lanhouin au Bénin. Thèse de Doctorat, Université technologique d'Etat de Krasnodar, Russie, p. 129.

Essuman KM. 1992. Le poisson fermenté en Afrique : traitement, commercialisation et consommation. FAO. Doc. Tech. Pêches, 329. Rome.

Guiraud J, Galzy P. 1980. L'Analyse Microbiologique dans les Industries Alimentaires. Collection Génie Alimentaire. Editions de l'Usine Nouvelle : Paris.

Martin PG. 1979. Manuals of Foods Quality Control 3. Commodities. FAO, Rome.

Pearson D. 1976. The Chemical Analysis of Foods (7th edn). A. Churchill Livingstone: Edinburg; London; New York.

Tohouégnon CP, Diei Y, Dehy L. 1997. Amélioration de la méthode traditionnelle de fabrication du 'lanhouin', au Bénin. Rapport technique, Direction de la Pêche, Cotonou. 